\title{
Assessment of myocardial extracellular volume on body computed tomography in breast cancer patients treated with anthracyclines
}

\author{
Caterina Beatrice Monti ${ }^{1}$, Moreno Zanardo ${ }^{1}$, Tommaso Bosetti ${ }^{2}$, Marco $\mathrm{Ali}^{3,4}$, Elena De Benedictis ${ }^{5}$, \\ Alberto Luporini ${ }^{6}$, Francesco Secchi ${ }^{1,4}$, Francesco Sardanelli ${ }^{1,4}$ \\ ${ }^{1}$ Department of Biomedical Sciences for Health, Università degli Studi di Milano, Via Mangiagalli 31, 20133 Milan, Italy; ${ }^{2}$ Medicine and Surgery \\ School, Università degli Studi di Milano, Via Festa del Perdono 7, 20122 Milan, Italy; ${ }^{3}$ Unit of Diagnostic Imaging and Stereotactic Radiosurgery, \\ CDI Centro Diagnostico Italiano S.p.A., Via Saint Bon 20, 20147 Milan, Italy; ${ }^{4}$ Unit of Radiology, ${ }^{5}$ Unit of Oncology I, ${ }^{6}$ Unit of Oncology II, \\ IRCCS Policlinico San Donato, Via Morandi 30, 20097 San Donato Milanese, Italy
}

Correspondence to: Dr. Moreno Zanardo. Department of Biomedical Sciences for Health, Università degli Studi di Milano, Via Mangiagalli 31 , 20133 Milan, Italy. Email: moreno.zanardo@unimi.it.

Background: Cancer treatment with anthracyclines may lead to an increased incidence of cardiac disease due to cardiotoxicity, as they may cause irreversible myocardial fibrosis. So far, the proposed methods for screening patients for cardiotoxicity have led to only limited success, while the analysis of myocardial extracellular volume (mECV) at cardiac magnetic resonance (CMR) has shown promising results, albeit requiring a dedicated exam. Recent studies have found strong correlations between mECV values obtained through computed tomography (CT), and those derived from CMR. Thus, our purpose was to evaluate the feasibility of estimating mECV on thoracic contrast-enhanced CT performed for staging or follow-up in breast cancer patients treated with anthracyclines, and, if feasible, to assess if a rise in mECV is associated with chemotherapy, and persistent over time.

Methods: After ethics committee approval, female patients with breast cancer who had undergone at least 2 staging or follow-up CT examinations at our institution, one before and one shortly after the end of chemotherapy including anthracyclines were retrospectively evaluated. Patients without available haematocrit, with artefacts in CT images, or who had undergone radiation therapy of the left breast were excluded. Follow-up CT examinations at longer time intervals were also analysed, when available. mECV was calculated on scans obtained at 1 , and $7 \mathrm{~min}$ after contrast injection.

Results: Thirty-two female patients (aged $57 \pm 13$ years) with pre-treatment haematocrit $38 \% \pm 4 \%$, and ejection fraction $64 \% \pm 6 \%$ were analysed. Pre-treatment $\mathrm{mECV}$ was $27.0 \% \pm 2.9 \%$ at $1 \mathrm{~min}$, and $26.4 \% \pm 3.8 \%$ at $7 \mathrm{~min}$, similar to values reported for normal subjects in the literature. Post-treatment mECV (median interval: 89 days after treatment) was $31.1 \% \pm 4.9 \%$, and $30.0 \% \pm 5.1 \%$, respectively, values significantly higher than pre-treatment values at all times $(\mathrm{P}<0.005)$. mECV at follow-up (median interval: 135 days after post-treatment $\mathrm{CT}$ ) was $31.0 \% \pm 4.5 \%$, and $27.7 \% \pm 3.7 \%$, respectively, without significant differences $(\mathrm{P}>0.548)$ when compared to post-treatment values.

Conclusions: mECV values from contrast-enhanced CT scans could play a role in the assessment of myocardial condition in breast cancer patients undergoing anthracycline-based chemotherapy. CT-derived ECV could be an imaging biomarker for the monitoring of therapy-related cardiotoxicity, allowing for potential secondary prevention of cardiac damage, using data derived from an examination that could be already part of patients' clinical workflow.

Keywords: Extracellular space; myocardium; anthracyclines; cardiotoxicity; tomography, X-ray computed

Submitted Sep 20, 2019. Accepted for publication Mar 31, 2020.

doi: $10.21037 /$ qims.2020.04.05

View this article at: http://dx.doi.org/10.21037/qims.2020.04.05 


\section{Introduction}

Breast cancer (BC) is the most common cancer in women, with 523,000 new cases and 138,000 deaths in Europe in 2018 (1). The average woman has a 1 in 8 chance of developing $\mathrm{BC}$ in their lifetime (2).

Among BC patients, anthracyclines represent a treatment mainstay, used to manage both early state and metastatic disease $(3,4)$. However, anthracyclines may cause cardiotoxicity expressed as an increase in myocardial fibrosis, with a cumulative effect (thought to be irreversible), and no options for a safe dosage (5). Fibrosis may lead to asymptomatic left ventricular ejection fraction (LVEF) drop, hypertension, arrhythmias, QTc-interval prolongation, overt heart failure or myocardial ischemia $(6,7)$.

Even though different potential biomarkers for chemotherapy cardiotoxicity have been proposed, none has so far been proved to be able to timely detect subclinical myocardial changes (8). Therefore, there is a need for reliable and sensitive biomarkers, which could allow an early detection of myocardial changes, to guide physicians in undertaking prevention of cardiac dysfunction in women at risk (9). Detecting cardiotoxicity in a timely manner would in fact allow physicians to undertake preventive measures such as the administration of Dexrazoxane (10).

Extracellular volume (ECV) is an imaging biomarker which reflects the percentage of myocardium not constituted by cells and can increase in presence of fibrosis or pathological depositions such as those of amyloidosis $(11,12)$. When assessed by cardiac magnetic resonance (CMR) through dedicated pulse sequences, ECV has been shown to correlate well with histological collagen volume fraction, its increase representing a risk factor for heart failure or cardiac-related death $(13,14)$. Normal value ranges have been proposed as a reference for CMR-derived ECV $(15,16)$.

Over the last few years, ECV calculation by computed tomography (CT) has been also proposed (17). So far, CTderived ECV has been assessed through dedicated cardiac CT examinations (17). Bandula et al. (18) have shown that CT-derived ECV has a robust correlation with both CMRderived ECV and histological findings.

In the guidelines for clinical management of $\mathrm{BC}$, contrast-enhanced chest $\mathrm{CT}$ is recommended for staging or restaging at all clinical stages if pulmonary involvement is suspected, and it is always recommended for clinical stage III patients, albeit not being recommended for screening of disease recurrence in asymptomatic patients (3). Notably, a standard contrast-enhanced chest CT allows for the visualization of the heart, and oncologic CT protocols may include a non-contrast scan, an arterial phase scan, a portalvenous phase scan at $1 \mathrm{~min}$ after contrast administration and delayed-phase scans from 3 min after contrast administration (19), either on both chest and abdomen or on the abdomen only. The left ventricle is usually at least partially visible even in abdominal scans.

A key consideration in this context is given by the major relevance of cardiac disease in BC survivors. Older women diagnosed with $\mathrm{BC}$ are almost equally likely to die because of $\mathrm{BC}$ as they are to die because of cardiovascular disease (20).

Thus, the aims of our study were: (I) to assess whether $\mathrm{BC}$ treatment with anthracyclines was associated to an increased ECV even in the absence of clinical, echocardiographic, or electrocardiographic signs of cardiac damage; and (II) to appraise whether any increase in myocardial ECV remained unchanged at follow-up.

\section{Methods}

\section{Ethical statement and study design}

The local Ethics Committee approved this study (Ethics Committee of San Raffaele Clinical Research Hospital; protocol code "CardioRetro", number 122/int/2017; approved on September 14th, 2017 and amended on May 10th, 2018). Due to the retrospective nature of this study, specific informed consent was waived.

\section{Study population}

All patients who had undergone anthracycline treatment for BC at our institution from May 2012 to May 2018, whose contrast-enhanced CT examinations prior to and no longer than 5 months after the end of anthracycline treatment were available in our database, were included. All patients had been treated according to guidelines (3), and anthracycline treatment had been administered in intravenous bolus for $5 \mathrm{~min}$, once every three weeks. Treatment would have been postponed for one week if neutrophils were under $1,500 / \mathrm{mm}^{3}$, and the dose would have been reduced to $75 \%$ in case of febrile neutropenia. As per clinical recommendations, CT examinations were performed for staging and re-staging patients advanced stage disease, or in patients with symptoms of potential pulmonary involvement.

Exclusion criteria were the lack of haematocrit value obtained from 4 weeks before to the date of the pre- 
treatment CT examination, and further than 4 weeks for subsequent examinations or intercurrent surgeries or blood transfusions in the time between haematocrit measurements and CT scans, as well as incomplete heart scans or the presence of artefacts in the longitudinal view of the heart in the unenhanced scan or in any of the contrast-enhanced scans. Moreover, patients who had undergone radiation therapy for cancer in the left breast were excluded due to its potential confounding cardiotoxicity (21). Patients also underwent an electrocardiogram and echocardiography, and patients with underlying cardiac pathology were excluded.

For follow-up assessment, we considered all patients having another contrast-enhanced CT examination acquired at least 5 months after the end of anthracycline chemotherapy and at least 3 months after the first followup CT, so that any leftover myocardial oedema should have resolved (22), again excluding those who had undergone radiation therapy in the left breast or other cardiotoxic chemotherapy or targeted therapy (i.e., trastuzumab), and those who either lacked haematocrit values no further than 4 weeks from CT examination, or underwent surgery or blood transfusion in the time between haematocrit measurements and CT scans.

\section{CT acquisition protocol}

Patients were studied using 1 of 2 CT scanners, one 64-slice unit and one 16-slice unit. For the 64-slice CT unit (Somatom Definition, Siemens), the following technical parameters were used: $120 \mathrm{kVp}$; tube current from 100 to $200 \mathrm{mAs}$, depending on automatic exposure control system (CARE Dose 4D, Siemens); rotation time $0.5 \mathrm{~s}$; pitch 1; kernel reconstruction technique B30f medium smooth. For the 16-slice CT unit (Emotion 16, Siemens), the following technical parameters were used: $130 \mathrm{kVp}$; tube current from 100 to $200 \mathrm{mAs}$, depending on automatic exposure control system (CARE Dose 4D, Siemens), rotation time $0.5 \mathrm{~s}$; pitch 1, kernel reconstruction technique B30f medium smooth. All patients underwent total body CT scans including abdomen, at least part of the chest and the heart, and head. Slice thickness was $5 \mathrm{~mm}$.

An iodinated contrast agent (iopamidol, Iopamiro 370; $370 \mathrm{mg} \mathrm{I} / \mathrm{mL}$; Bracco Imaging) was injected intravenously at a dose of $1.2 \mathrm{~mL} / \mathrm{kg}$ through a 20 -gauge needle using an automatic injector (EmpowerCTA Contrast Injection System, Bracco Imaging) at the rate of $3 \mathrm{~mL} / \mathrm{s}$, followed by $50 \mathrm{~mL}$ of saline solution at the same rate.

Scan delay was determined using an automated triggering hardware and a dedicated software (Bolus Tracking, Siemens Healthineers, Erlangen, Germany). Specifically, low-dose monitor images were obtained in a single axial slice of the aorta after contrast injection. After obtaining a pre-contrast scan, when the descending aorta enhanced more than 100 Hounsfield unit (HU), the first contrast-enhanced series was acquired (arterial phase, about 15-18 s after contrast injection). Afterwards, additional scans were acquired for obtaining portal venous (1 min after contrast injection) and delayed phase at $7 \mathrm{~min}$ after contrast injection.

Patients were instructed to hold their breath during acquisition and craniocaudal (top-down) scanning direction was adopted for all scans. Radiation doses were reported using dose-length product (DLP), expressed in $\mathrm{mGy} \times \mathrm{cm}$, and the total DLP value per patient were extracted.

\section{Image analysis}

All images were reviewed by a reader with a 2 -year experience in body CT, and half of them, randomly chosen, were reviewed by another reader with a 3-year experience. First, the observer chose the best slice to visualize a longitudinal view of the cardiac chambers. Then, measurements were obtained by manually placing a round region of interest (ROI) as large as possible in the mid-level ventricular septum and a second ROI in the intraventricular blood pool at the same level, again as large as possible but avoiding papillary muscles (based on contrast-enhanced scans). This was done on scans acquired at 1, and $7 \mathrm{~min}$, as shown in Figure 1 . Concerning unenhanced scans, ROI placements were copied from the $1 \mathrm{~min}$ scan, and placed in the same positions, then performing subtle adjustments considering density, as the myocardium is slightly denser than the blood on basal scans, as shown in Figure 2. As scans were not synchronized to an electrocardiogram, while placing ROIs, cardiac movement was considered: ROIs were mainly placed in the middle of the septum, excluding the boundaries close to the intraventricular blood pool.

Septal ECV was calculated using the following formula, according to Miller et al. (14):

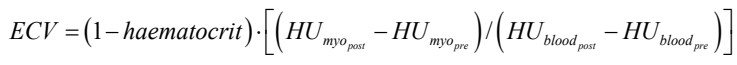

where: $m y o=$ myocardium; pre $=$ pre-contrast post $=$ post contrast.

Moreover, we also recorded standard deviations (SD) for each ROI placed in the myocardium, and SD of background air as a measure of background noise, to appraise a potential contamination of measurements of myocardial HUs by 

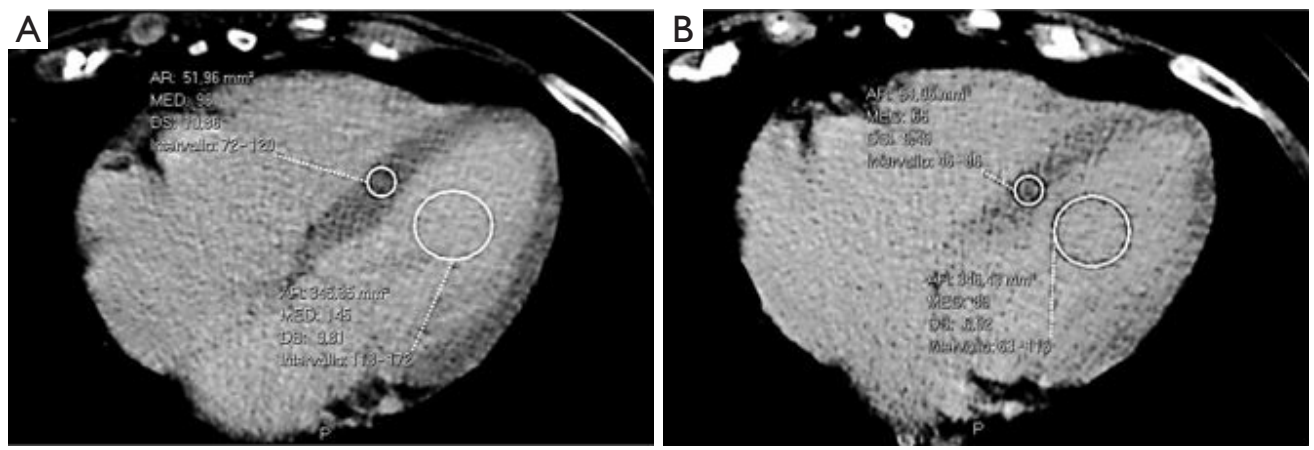

Figure 1 Examples of regions of interest used for extracellular volume estimation, in computed tomography scans in a 77-year-old woman: (A) at $1 \mathrm{~min}$ and (B) at $7 \mathrm{~min}$.
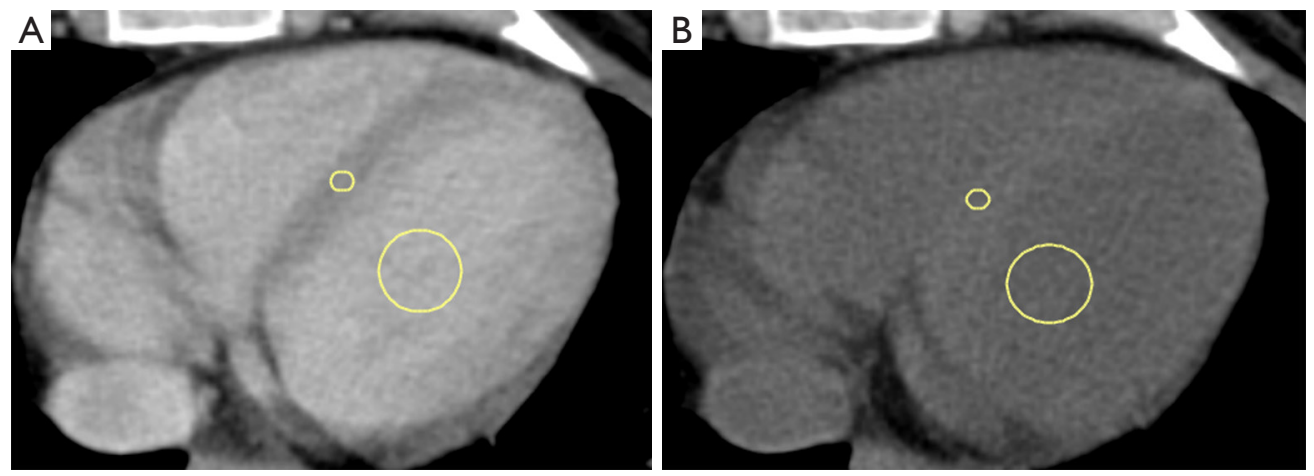

Figure 2 Depiction of region of interest placement on basal and 1 min post-contrast scan in a 30-year-old female patient. The regions of interest were first placed in the 1 min scan, where the myocardium and blood were more clearly discernible, and then copied in the same positions in the basal scan.

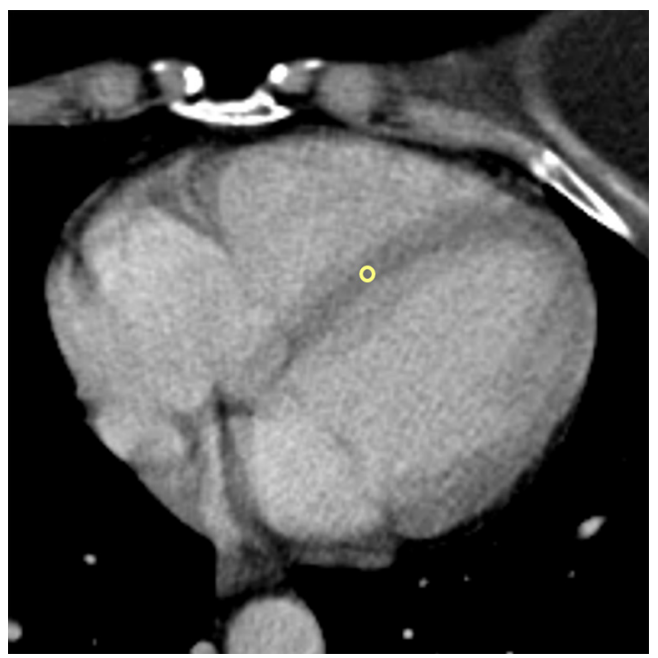

Figure 3 Region of interest placement in a 62-year-old female patient, presenting motion artifacts involving the heart. The region of interest was placed in the mid-septum, excluding the areas closer to the ventricular cavity, where blood contamination was expected. motion artifacts and subsequent presence of blood in the ROI. An example of ROI placement in presence of motion artifacts is shown in Figure 3.

\section{Statistical analysis}

Continuous variables were tested for normality with the Shapiro-Wilk test. Parametric data were reported as mean $\pm \mathrm{SD}$, while non-parametric data were reported as median and interquartile range (IQR).

Inter-reader reproducibility of CT-derived ECV was assessed in a subset of randomly chosen patients on the pretreatment scans, with a two-way, mixed-effects interclass correlation coefficient (ICC). ICCs were reported and interpreted according to Koo and Li (23).

Correlations were studied with Pearson $r$ or Spearman $\rho$ with regards to data normality. Correlation coefficients were interpreted according to Evans (24). When correlations between 
ECV at different timings were present, Bland-Altman analyses were conducted to evaluate data consistency. Differences were appraised with $t$-test when variables were normal, or with Wilcoxon's test when variables were not normal.

Potential contamination of the HUs of the myocardial ROIs was studied comparing the ratio between myocardial HU SD and background noise, represented by the SD of background air, to 1, with a one-sample parametric or nonparametric test, $t$-test or Mann-Whitney $\mathrm{U}$ respectively, with regards to data distribution.

Statistical analysis was performed with SPSS v20 (IBM SPSS Inc.). $\mathrm{P}$ values $<0.05$ were considered as significant.

\section{Results}

\section{Study population}

Based on the availability of studies, 42 female patients were originally considered for analysis. After the application of exclusion criteria, our population for ECV assessment before/after treatment was composed by 32 patients. From the initial population, 8 patients were excluded due to haematocrit values being out of the desired time interval, 1 due to incomplete CT scans, and 1 since she underwent left radiation therapy. For ECV follow-up, 13 patients were excluded for the lack of an additional CT examination at appropriate timing and 8 since they underwent left radiation therapy between the two CT examinations, leading to 32 patients being available for ECV comparison between pre- and post-chemotherapy, and 11 patients available for follow-up analysis (Figure 4).

Age at the first CT was $57 \pm 13$ years; 29 patients had an infiltrating ductal carcinoma, 2 a poorly differentiated carcinoma, and 1 a neuroendocrine breast carcinoma. All patients had stage II or higher disease. Mean haematocrit before chemotherapy was $38 \% \pm 4 \%$, while electrocardiogram and echocardiography showed no abnormal findings, with a mean LVEF at echocardiography of $64 \% \pm 6 \%$. Baseline characteristics of the study population are presented in Table 1. No subject showed cardiotoxicity during or after anthracycline treatment, defined as a decrease in LVEF of more than $10 \%$ to below the lower limit of normal (25), and no subject experienced symptoms of cardiac toxicity either. All patients underwent chemotherapy with dosages adjusted to body weight and body surface area according to clinical guidelines (3). No patient received reduced doses or less treatment cycles due to toxicity. Twenty-six patients received Epirubicin at $90 \mathrm{mg} / \mathrm{m}^{2}$ per cycle (total 4 cycles) for a total dose of $360 \mathrm{mg} / \mathrm{m}^{2} ; 4$ patients received mitoxantrone at $10 \mathrm{mg} / \mathrm{m}^{2}$ per cycle (total 4 cycles) for a total dose of $40 \mathrm{mg} / \mathrm{m}^{2}$; and 2 received dosages of $60 \mathrm{mg} / \mathrm{m}^{2}$ of Adriamycin per cycle (total 4 cycles) for a total dose of $240 \mathrm{mg} / \mathrm{m}^{2}$. Out of 75 total CT examinations (32 pre-treatment, 32 post-treatment and 11 at follow-up), all 75 examinations included the abdomen, part of the chest and the heart, and the head. DLP were highly variable due to the different $\mathrm{CT}$ acquisition protocols (chest or abdomen) and total scan volume: mean and SD were $3,847 \pm 934 \mathrm{mGy} \times \mathrm{cm}$, with a minimum DLP value of 2,093 $\mathrm{mGy} \times \mathrm{cm}$ and a maximum value of 5,680 $\mathrm{mGy} \times \mathrm{cm}$.

\section{Pre-treatment myocardial ECV and reproducibility}

Thirty-two patients were assessed for pre-treatment analyses. Pre-treatment ECV was $27.0 \% \pm 2.9 \%$ (mean \pm $\mathrm{SD})$ at $1 \mathrm{~min}$, and $26.4 \% \pm 3.8 \%$ at $7 \mathrm{~min}$ (Figure 5). Interreader reproducibility was good at all scanning times: ICC was 0.78 [95\% confidence interval (CI): 0.39-0.92] at $1 \mathrm{~min}$, and 0.80 (95\% CI: $0.42-0.93)$ at $7 \mathrm{~min}$.

Pre-treatment ECV at 1 min showed significant correlations with pre-treatment ECV 7 min $(r=0.743, \mathrm{P}<0.001)$. BlandAltman analysis between ECV at 1 and 7 min showed a bias of $0.91 \%$ and a coefficient of repeatability of $12 \%$.

\section{Myocardial ECV after therapy}

The median interval between the end of chemotherapy and the first CT after treatment was 89 days (IQR, 23-127 days); 32 patients were analysed for post-treatment analysis.

Post-treatment haematocrit was $35 \% \pm 3 \%($ mean $\pm \mathrm{SD})$, with a significant decrease from pre-treatment values $(\mathrm{P}=0.001)$. Post-treatment ECV was $31.1 \% \pm 4.9 \%$ at $1 \mathrm{~min}$, and $30.0 \% \pm 5.1 \%$ at $7 \mathrm{~min}$, values significantly higher than those obtained before therapy at all scan times, with $\mathrm{P}<0.001$ at $1 \mathrm{~min}$, and $\mathrm{P}=0.002$ at $7 \mathrm{~min}$. The average increase in $\mathrm{ECV}$ was $4.1 \%$ (IQR, 1.2-6.5\%), and $2.7 \%$ (IQR, 0.2-5.9\%), respectively (see Figure 5).

\section{Follow-up}

The median interval from post-treatment CT to follow-up CT was 135 days (IQR, 116-159 days), the median interval from pre-treatment CT to follow-up CT was 312 days (IQR, 242-401 days); 11 patients were analysed for followup analyses.

At follow-up, haematocrit was $35 \% \pm 3 \%($ mean $\pm \mathrm{SD})$. 


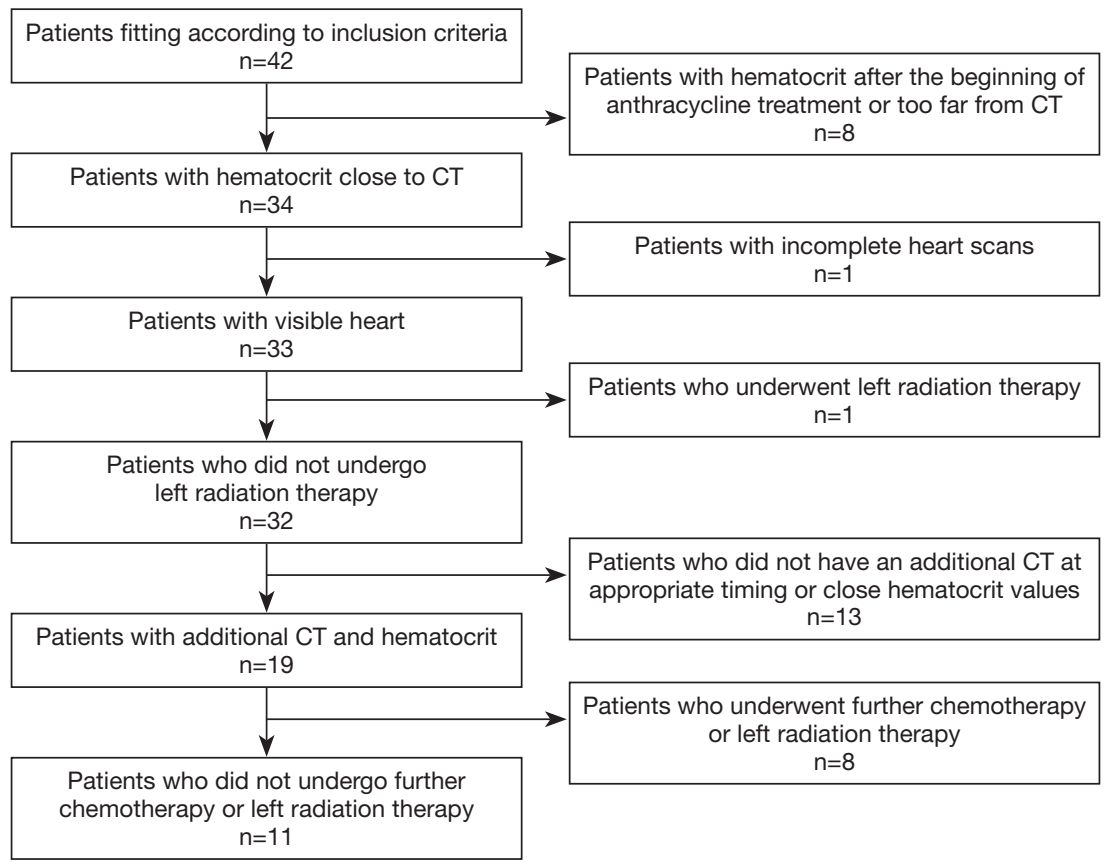

Figure 4 Flowchart describing patient selection. From the initial population of 42 women complying to inclusion criteria, 10 patients were excluded due to: lack of close haematocrit values $(n=8)$; pre-treatment scan not including any analysable portion of the heart ( $\mathrm{n}=1$ ); intercurrent radiation therapy to the left breast $(n=1)$. After the post-treatment evaluation, 21 additional patients were excluded due to: lack of a further computed tomography examination or close haematocrit $(n=13)$; intercurrent radiation therapy to the left breast $(n=8)$.

Table 1 Baseline characteristics of the study population

\begin{tabular}{lc}
\hline Variable & Value (N=32) \\
\hline Age (years), mean \pm SD & $57 \pm 13$ \\
Type of carcinoma & 29 \\
Infiltrating ductal & 2 \\
Poorly differentiated & 1 \\
Neuroendocrine & \\
Disease stage & 10 \\
II & 13 \\
III & 9 \\
IV & $64 \pm 6$ \\
LVEF (\%), mean \pm SD & $38 \pm 4$ \\
Hematocrit (\%), mean \pm SD & \\
\hline
\end{tabular}

SD, standard deviation; LVEF, left ventricular ejection fraction.

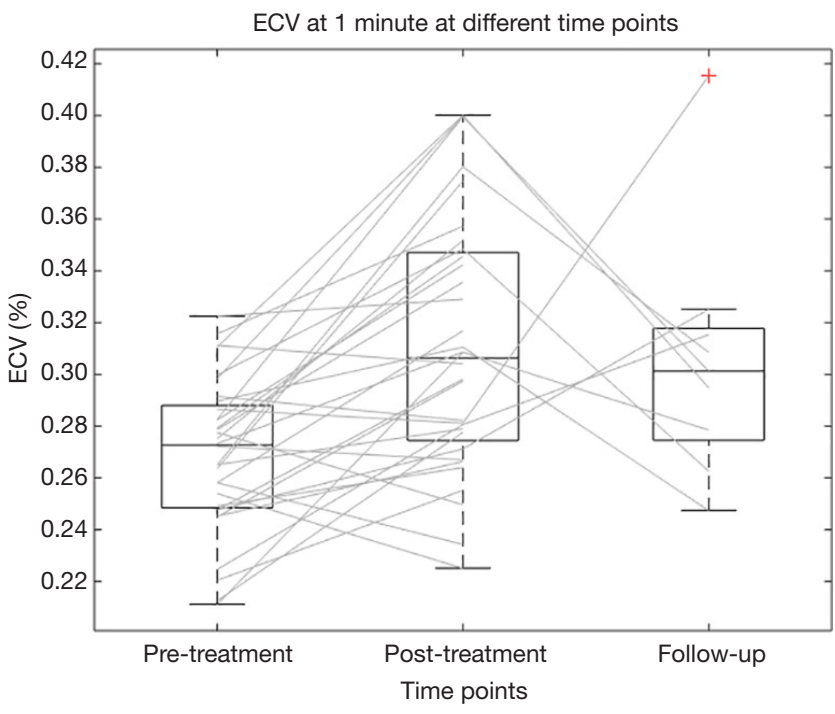

Figure 5 Boxplot depicting values of myocardial extracellular volume $(\mathrm{ECV})$ at $1 \mathrm{~min}$ at different time points, namely before treatment, after treatment and at follow-up. Individual subjects ECV variations are represented by connecting lines. 
The ECV was $31.0 \% \pm 4.5 \%$ at $1 \mathrm{~min}$, and $27.7 \% \pm 3.7 \%$ at $7 \mathrm{~min}$. There were no significant differences between follow-up and post-treatment ECV at $1 \mathrm{~min}(\mathrm{P}=0.669)$, and 7 min $(\mathrm{P}=0.549)$. Follow-up ECV was significantly higher than pre-treatment $\mathrm{ECV}$ at $1 \mathrm{~min}(\mathrm{P}=0.002)$, but not at $7 \mathrm{~min}(\mathrm{P}=0.288)$ (see Figure 5).

\section{Image quality of the myocardium}

The median SD of myocardial ROIs was $7.25 \mathrm{HU}$ (IQR, 6.26-8.60 HU) for the basal scan, 8.62 HU (IQR, 6.74-9.91 HU) at $1 \mathrm{~min}$, and $8.73 \mathrm{HU}$ (IQR, 7.06-10.11 HU) at $7 \mathrm{~min}$. The median SD of the background was $8.45 \mathrm{HU}$ (IQR, 5.98-11.75 HU) for the basal scan, $8.98 \mathrm{HU}$ (IQR, 6.33-11.63 $\mathrm{HU}$ ) at $1 \mathrm{~min}$, and $9.21 \mathrm{HU}(\mathrm{IQR}, 6.79-11.76 \mathrm{HU}$ ) at $7 \mathrm{~min}$. The median ratios between SD of the myocardium and background were 0.92 (IQR, 0.66-1.18) for the basal scan, 0.96 (IQR, 0.75-1.23) at $1 \mathrm{~min}$, and 0.96 (IQR, $0.75-1.24$ ) at $7 \mathrm{~min}$, and neither ratio was significantly different from $1(\mathrm{P}=0.187, \mathrm{P}=0.921$, and $\mathrm{P}=0.817$, respectively).

\section{Discussion}

In this study we demonstrated the feasibility of using ECV from CT scans as a biomarker of myocardial condition in oncologic patients undergoing potentially cardiotoxic chemotherapy.

Our relatively small population can be considered representative of the average advanced $\mathrm{BC}$ patients in terms of age $(57 \pm 13$ years). In fact, women are diagnosed with $\mathrm{BC}$ at a median age of 61 years, and patients with metastatic disease undergoing chemotherapy tend to be younger $(26,27)$. Considering that none of our patients was ever reported to have cardiac disease, with normal electrocardiogram and LVEF at echocardiography, we can assume for their hearts to be healthy prior to chemotherapy.

Our pre-treatment myocardial ECV values are in agreement with those reported by Kurita et al. in 14 healthy women at $7 \mathrm{~min}$ post-contrast injection (28), demonstrating that ECV values obtained by body CT are consistent with those obtained by dedicated cardiac CT. Our higher limit for ECV range compared to the study by Kurita et al. may be due to their stricter criteria for cardiac health condition, for instance excluding all subjects with a calcium scoring $\geq 100$. The good inter-reader reproducibility at all scanning times suggests that CT-derived ECV should be not only accurate but also precise. The positive correlations between
ECV measured at different times may indicate that all values are related to the extent of ECV.

Patients were treated with anthracyclines according to current guidelines (3). Although they were treated with different drugs, all anthracycline-based drugs have been proven to be cardiotoxic at such doses (29). The significant difference in ECV between pre- and post- treatment can be explained by the known mechanisms of anthracycline cardiotoxicity, classified as type I damage according to Ewer et al. (30), leading to cell apoptosis and necrosis, thus to oedema at acute phase, and consequent fibrosis, both determining an increase in ECV (12).

The average increase in ECV we found in this study presented a wide distribution of values. This suggests that patients may respond differently to anthracycline treatment in terms of myocardial oedema and fibrosis, with some experiencing a steep increase most likely due to oedema and fibrosis following necrosis, and others maintaining a constant ECV. In fact, different predictors of anthracycline cardiotoxicity have been reported (31), and patients could react differently due to pre-existing conditions such as diabetes, overweight, smoking, or sedentary life (32). An earlier identification of patients more susceptible to anthracycline cardiotoxicity may help adopting preventive actions, e.g. the administration of cardioprotectors such as Dexrazoxane (10).

Our follow-up results confirmed that CT-derived ECV reflects anthracycline cardiotoxicity. Of note, type I cardiotoxicity is considered as irreversible (30). Thus, since our ECV values did not revert to pre-treatment levels months after the end of therapy, we may hypothesize for this ECV increase to be permanent or at least markedly prolonged. This phenomenon resulted to be clear for ECV values obtained at $1 \mathrm{~min}$ after contrast injection. For ECV values obtained at $7 \mathrm{~min}$, we observed a highly significant increase from pre-treatment $(26.4 \% \pm 3.8 \%)$ to posttreatment $(30.0 \% \pm 5.1 \%)$ and a reduction to $27.7 \% \pm 3.7 \%$ at follow-up, the last value being not significant different from that of both pre-treatment and post-treatment. This plays in favour of a partial revert of ECV to pre-treatment values but also opens the issue of which timing after contrast injection is optimal for estimating ECV using CT scans before/after the injection of iodinated contrast material, to be investigated in future researches. Our timing of $7 \mathrm{~min}$ is close to the acquisition timing of $5 \mathrm{~min}$ post-contrast injection which was found to be ideal for calculating ECV on CT scans by Treibel et al. (33). Moreover, one study by Hamdy et al. (34) showed that delay times of 3, 5, and $7 \mathrm{~min}$ 
after contrast injection provided compatible ECV values, and our strong correlation and good agreement between values obtained at 1 and $7 \mathrm{~min}$ suggests the presence of a common denominator which may be evaluated at both times. The presence of various scans acquired at different times stems from the use of a past imaging protocol, including more scans than currently recommended. We thus utilized the available scans at different timings to evaluate the feasibility of calculating ECV from contrastenhanced CT.

The detection of chemotherapy cardiotoxicity has already been proposed among the possible clinical uses of ECV (35). Indeed, Jordan et al. (36) and Neilan et al. (37), have assessed ECV as a biomarker of anthracycline cardiotoxicity using CMR T1-mapping before/after the administration of gadolinium-based contrast agent. Both studies concluded that years after the end of treatment with anthracyclines, ECV was still elevated compared to patients not treated with cardiotoxic drugs, in agreement with our findings at follow-up. However, we should consider that both studies assessed myocardial ECV via CMR, which is a relatively high-cost examination, not included into the assessment of $\mathrm{BC}$ patients undergoing chemotherapy. In addition, CMR is more time-consuming than $\mathrm{CT}$, it has additional contraindications such as obesity or claustrophobia, and concerns about the safety of repeated injections of gadolinium-based contrast agents have been raised (38).

DLP was elevated and highly variable considering that patients underwent different CT protocols on two different CT scanners, one of them being an old 16-row unit, and all patients performed a total body CT scan (head, chest, abdomen and pelvis), reporting in these cases high values of DLP, however still under the DRL reverences values (39). Nevertheless, an ideal protocol for the analysis of ECV on CT would include a basal acquisition on the heart, and venous phase scan, totalling around $0.3-0.4 \mathrm{mSv}$ and 4-5 $\mathrm{mSv}$ respectively (40).

\section{Limitations}

This study has limitations. First, it is retrospective and monocentric, and thus a non-negligible number of subjects were not included due to the lack of contemporary haematocrit values, scans not sufficiently including the heart, or missing CT examinations. These factors reduced the sample size, limiting the statistical power, as discussed for ECV values obtained at $7 \mathrm{~min}$ at follow-up. Moreover, haematocrit measurement times were not standardized.
However, the intrinsic variability of haematocrit is estimated to be around $10 \%$ as observed by Thirup (41), i.e., 0.42 to 0.47 , in the absence of significant events as bleeding or treatment suppressing the bone marrow. Patients who experienced such events were excluded from the study as per exclusion criteria, and while their timings of haematocrit measurement remain indeed varied, we may estimate such variation to be small and follow a random pattern, leading to a casual distribution which would not affect the eventual mean over the whole sample. Nevertheless, this initial experience showed the clinical feasibility of myocardial ECV estimate on oncologic CT scans. Moreover, while CT is not recommended as a screening for disease recurrence, or as a staging and restaging imaging technique for asymptomatic, early stage $\mathrm{BC}$, a non-negligible number of patients undergoing heavily cardiotoxic treatments has advanced disease, and CT may indeed be part of their clinical workflow. Second, we used two different CT units (64- or 16-slice equipment), and our patients had undergone imaging according to a past protocol, including scans that are not currently recommended for disease staging and re-staging. Concerning the CT unit, being densitometry measured on CT scans always standardized to the water density (42), the measurements should be considered as reliable on both units. Regarding the use of an obsolete protocol, the main aim of our work was to evaluate feasibility of ECV from contrast-enhanced CT, and its results do not imply that a proposed prospective protocol for ECV assessment would need to include all the acquisitions that were performed on our patients. In fact, given the results obtained in the study, ECV could likely be calculated only using the $1 \mathrm{~min}$ scan, representing venous phase and thus recommended in staging and re-staging protocols, and one basal slice acquired on the heart to obtain pre-contrast $\mathrm{HU}$ values. Third, image time points were not standardized. However, studies on anthracycline cardiotoxicity have shown that it is characterized by necrosis and subsequent fibrosis (30), thus, knowing that the insult on patients' hearts ceased with the end of chemotherapy, we may assume oedema to be resolved by 312 days. Therefore, we may assume that our follow-up data reflects irreversible fibrosis. Fourth, our images were not triggered ad not synchronized to the cardiac cycle. However, we performed measurements to mid-septum, thus reducing the impact of this limitation. Moreover, we appraised potential contamination of ROI values by motion artifacts and presence of blood, by analysing the ratio between myocardial SD and background 
noise and tested the hypothesis that such ratio was equal to one, signifying that the two variables were equal and thus all the variation in myocardial HUs was due to background noise. We found that for every scan timepoint, we could not reject the null hypothesis that variation in myocardial HUs was compatible with background noise $(\mathrm{P}=0.187,0.921$ and 0.817 for basal, $1-$ and $7-\mathrm{min}$ scans respectively). Another limitation is the lack of a control group for ECV comparisons, and of a reference standard for our measurements of ECV via contrast-enhanced CT scans. However, the former issue is due to the fact that it is difficult to include in a retrospective study patients with $\mathrm{BC}$ who underwent $\mathrm{CT}$ and did not undergo treatments that may cause cardiotoxicity, as even trastuzumab or radiation therapy may bear cardiac-related adverse effects. Thus, reference values would need to be established via prospective studies before ECV assessment may be included in clinical practice.

In conclusion, we showed that myocardial ECV can be estimated on CT scans in BC patients undergoing anthracycline-based treatment, and that a significant ECV increase can be appreciated after treatment. In a clinical perspective, as survival rates for patients diagnosed with BC constantly increase, detection of toxicities related to treatments become a crucial issue (33). In this setting, CT-derived ECV could be an imaging biomarker for the monitoring of therapy-related cardiotoxicity, allowing for potential secondary prevention of cardiac damage, using data derived from an examination that could be part of patients' clinical workflow. Prospective studies are warranted to define the role of CT-derived myocardial $\mathrm{ECV}$ in BC patients.

\section{Acknowledgments}

We thank all the personnel of the IRCCS Policlinico San Donato for their support.

Funding: This work was supported by local research funds of IRCCS Policlinico San Donato, a Clinical Research Hospital partially funded by the Italian Ministry of Health. This research received no specific grant from any funding agency in the public, commercial or not-for-profit sectors.

\section{Footnote}

Conflicts of Interest: All authors have completed the ICMJE uniform disclosure form (available at http://dx.doi. org/10.21037/qims.2020.04.05). FS reports he has received research grants from and he is member of the speakers' bureau for General Electric, Bayer, and Bracco; he is also member of the Bracco Advisory Group. The other authors have no conflicts of interest to declare.

Ethical Statement: The local Ethics Committee approved this study (Ethics Committee of San Raffaele Clinical Research Hospital; protocol code "CardioRetro", number 122/int/2017; approved on September 14th, 2017 and amended on May 10th, 2018). Due to the retrospective nature of this study, specific informed consent was waived.

Open Access Statement: This is an Open Access article distributed in accordance with the Creative Commons Attribution-NonCommercial-NoDerivs 4.0 International License (CC BY-NC-ND 4.0), which permits the noncommercial replication and distribution of the article with the strict proviso that no changes or edits are made and the original work is properly cited (including links to both the formal publication through the relevant DOI and the license). See: https://creativecommons.org/licenses/by-nc-nd/4.0/.

\section{References}

1. Ferlay J, Colombet M, Soerjomataram I, Dyba T, Randi G, Bettio M, Gavin A, Visser O, Bray F. Cancer incidence and mortality patterns in Europe: Estimates for 40 countries and 25 major cancers in 2018. Eur J Cancer 2018;103:356-87.

2. DeSantis C, Ma J, Bryan L, Jemal A. Breast cancer statistics, 2013. CA Cancer J Clin 2014;64:52-62.

3. Goetz MP, Gradishar WJ, Anderson BO, Abraham J, Aft R, Allison KH, Blair SL, Burstein HJ, Dang C, Elias AD, Farrar WB, Giordano SH, Goldstein LJ, Isakoff SJ, Lyons J, Marcom PK, Mayer IA, Moran MS, Mortimer J, O'Regan RM, Patel SA, Pierce LJ, Reed EC, Rugo HS, Sitapati A, Smith KL, Smith M Lou, Soliman H, Telli ML, Ward JH, Young JS, Shead DA, Kumar R. Breast Cancer, Version 3.2018. J Natl Compr Cancer Netw 2019;17:118-26.

4. MacKenzie JR. Complications of treatment of paediatric malignancies. Eur J Radiol 2001;37:109-19.

5. Ryberg M, Nielsen D, Cortese G, Nielsen G, Skovsgaard T, Andersen PK. New insight into epirubicin cardiac toxicity: Competing risks analysis of 1097 breast cancer patients. J Natl Cancer Inst 2008;100:1058-67.

6. Farhad H, Staziaki PV, Addison D, Coelho-Filho OR, Shah RV, Mitchell RN, Szilveszter B, Abbasi SA, Kwong 
RY, Scherrer-Crosbie M, Hoffmann U, Jerosch-Herold $M$, Neilan TG. Characterization of the Changes in Cardiac Structure and Function in Mice Treated With Anthracyclines Using Serial Cardiac Magnetic Resonance Imaging. Circ Cardiovasc Imaging 2016;9:e003584.

7. Ades F, Zardavas D, Pinto AC, Criscitiello C, Aftimos P, de Azambuja E. Cardiotoxicity of systemic agents used in breast cancer. Breast 2014;23:317-28.

8. Cardinale D, Colombo A, Bacchiani G, Tedeschi I, Meroni CA, Veglia F, Civelli M, Lamantia G, Colombo N, Curigliano G, Fiorentini C, Cipolla CM. Early detection of anthracycline cardiotoxicity and improvement with heart failure therapy. Circulation 2015;131:1981-8.

9. Irwin ML, Fabian C, McTiernan A. Risk reduction from weight management and physical activity interventions. Ganz PA, editor. Adv Exp Med Biol 2015;862:193-212.

10. Testore F, Milanese S, Ceste M, de Conciliis E, Parello G, Lanfranco C, Manfredi R, Ferrero G, Simoni C, Miglietta L, Ferro S, Giaretto L, Bosso G. Cardioprotective effect of dexrazoxane in patients with breast cancer treated with anthracyclines in adjuvant setting: a 10-year single institution experience. Am J Cardiovasc Drugs 2008;8:257-63.

11. Haaf P, Garg P, Messroghli DR, Broadbent DA, Greenwood JP, Plein S. Cardiac T1 mapping and extracellular volume (ECV) in clinical practice: a comprehensive review. J Cardiovasc Magn Reson 2016;18:89.

12. Cannaò PM, Altabella L, Petrini M, Alì M, Secchi F, Sardanelli F. Novel cardiac magnetic resonance biomarkers: native T1 and extracellular volume myocardial mapping. Eur Heart J Suppl 2016;18:E64-71.

13. Schelbert EB, Piehler KM, Zareba KM, Moon JC, Ugander M, Messroghli DR, Valeti US, Chang CCH, Shroff SG, Diez J, Miller CA, Schmitt M, Kellman P, Butler J, Gheorghiade M, Wong TC. Myocardial fibrosis quantified by extracellular volume is associated with subsequent hospitalization for heart failure, death, or both across the spectrum of ejection fraction and heart failure stage. J Am Heart Assoc 2015. doi: 10.1161/ JAHA.115.002613.

14. Miller CA, Naish JH, Bishop P, Coutts G, Clark D, Zhao S, Ray SG, Yonan N, Williams SG, Flett AS, Moon JC, Greiser A, Parker GJM, Schmitt M. Comprehensive validation of cardiovascular magnetic resonance techniques for the assessment of myocardial extracellular volume. Circ Cardiovasc Imaging 2013;6:373-83.

15. Gottbrecht M, Kramer CM, Salerno M. Native T1 and Extracellular Volume Measurements by Cardiac MRI in Healthy Adults: A Meta-Analysis. Radiology 2019;290:317-26.

16. Sardanelli F, Schiaffino S, Zanardo M, Secchi F, Cannaò PM, Ambrogi F, Di Leo G. Point estimate and reference normality interval of MRI-derived myocardial extracellular volume in healthy subjects: a systematic review and meta-analysis. Eur Radiol 2019;29:662033. Erratum in: Correction to: Point estimate and reference normality interval of MRI-derived myocardial extracellular volume in healthy subjects: a systematic review and meta-analysis. [Eur Radiol. 2019].

17. Nacif MS, Kawel N, Lee JJ, Chen X, Yao J, Zavodni A, Sibley CT, Lima JAC, Liu S, Bluemke DA. Interstitial myocardial fibrosis assessed as extracellular volume fraction with low-radiation-dose cardiac CT. Radiology 2012;264:876-83.

18. Bandula S, White SK, Flett AS, Lawrence D, Pugliese F, Ashworth MT, Punwani S, Taylor SA, Moon JC. Measurement of myocardial extracellular volume fraction by using equilibrium contrast-enhanced CT: validation against histologic findings. Radiology 2013;269:396-403.

19. Kuehl H, Veit P, Rosenbaum SJ, Bockisch A, Antoch G. Can PET/CT replace separate diagnostic CT for cancer imaging? Optimizing CT protocols for imaging cancers of the chest and abdomen. J Nucl Med 2007;48 Suppl 1:45S-57S.

20. Patnaik JL, Byers T, DiGuiseppi C, Dabelea D, Denberg TD. Cardiovascular disease competes with breast cancer as the leading cause of death for older females diagnosed with breast cancer: a retrospective cohort study. Breast Cancer Res 2011;13:R64.

21. Taylor C, McGale P, Brønnum D, Correa C, Cutter D, Duane FK, Gigante B, Jensen MB, Lorenzen E, Rahimi K, Wang Z, Darby SC, Hall P, Ewertz M. Cardiac structure injury after radiotherapy for breast cancer: cross-sectional study with individual patient data. J Clin Oncol 2018;36:2288-96.

22. Chatterjee K, Zhang J, Honbo N, Karliner JS. Doxorubicin cardiomyopathy. Cardiology 2010;115:155-62.

23. Koo TK, Li MY. A guideline of selecting and reporting intraclass correlation coefficients for reliability research. J Chiropr Med 2016;15:155-63.

24. Evans J. Straightforward statistics for behavioral sciences. Pacific Grove, California: Brooks/Cole Publishing, 1996.

25. Perez IE, Taveras Alam S, Hernandez GA, Sancassani R. Cancer Therapy-Related Cardiac Dysfunction: An 
Overview for the Clinician. Clin Med Insights Cardiol 2019; 13:1179546819866445.

26. Song QK, Li J, Huang R, Fan JH, Zheng RS, Zhang BN, Zhang B, Tang ZH, Xie XM, Yang HJ, He JJ, Li H, Li JY, Qiao YL, Chen WQ. Age of diagnosis of breast cancer in china: almost 10 years earlier than in the United States and the European union. Asian Pac J Cancer Prev 2014;15:10021-5.

27. Purushotham A, Shamil E, Cariati M, Agbaje O, Muhidin A, Gillett C, Mera A, Sivanadiyan K, Harries M, Sullivan R, Pinder SE, Garmo H, Holmberg L. Age at diagnosis and distant metastasis in breast cancer - A surprising inverse relationship. Eur J Cancer 2014;50:1697-705.

28. Kurita Y, Kitagawa K, Kurobe Y, Nakamori S, Nakajima H, Dohi K, Ito M, Sakuma H. Estimation of myocardial extracellular volume fraction with cardiac CT in subjects without clinical coronary artery disease: a feasibility study. J Cardiovasc Comput Tomogr 2016;10:237-41.

29. van Dalen EC, Michiels EM, Caron HN, Kremer LC. Different anthracycline derivates for reducing cardiotoxicity in cancer patients. Cochrane Database Syst Rev 2010;(3):CD005006.

30. Ewer MS, Lippman SM. Type II chemotherapy-related cardiac dysfunction: time to recognize a new entity. J Clin Oncol 2005;23:2900-2.

31. Lotrionte M, Biondi-Zoccai G, Abbate A, Lanzetta G, D’Ascenzo F, Malavasi V, Peruzzi M, Frati G, Palazzoni G. Review and meta-analysis of incidence and clinical predictors of anthracycline cardiotoxicity. Am J Cardiol 2013;112:1980-4.

32. Bulten BF, Verberne HJ, Bellersen L, Oyen WJG, Sabaté-Llobera A, Mavinkurve-Groothuis AMC, Kapusta L, van Laarhoven HWM, de Geus-Oei LF. Relationship of promising methods in the detection of anthracyclineinduced cardiotoxicity in breast cancer patients. Cancer Chemother Pharmacol 2015;76:957-67.

33. Treibel TA, Bandula S, Fontana M, White SK, Gilbertson JA, Herrey AS, Gillmore JD, Punwani S, Hawkins PN, Taylor SA, Moon JC. Extracellular volume quantification by dynamic equilibrium cardiac computed tomography in cardiac amyloidosis. J Cardiovasc Comput Tomogr 2015;9:585-92.

34. Hamdy A, Kitagawa K, Goto Y, Yamada A, Nakamura S, Takafuji M, Nagasawa N, Sakuma H. Comparison of the different imaging time points in delayed phase cardiac CT for myocardial scar assessment and extracellular volume fraction estimation in patients with old myocardial infarction. Int J Cardiovasc Imaging 2019;35:917-26.

35. Reiter U, Reiter C, Kräuter C, Fuchsjäger M, Reiter G. Cardiac magnetic resonance T1 mapping. Part 2: Diagnostic potential and applications. Eur J Radiol 2018;109:235-47.

36. Jordan JH, Vasu S, Morgan TM, D'Agostino RB, Meléndez GC, Hamilton CA, Arai AE, Liu S, Liu CY, Lima JAC, Bluemke DA, Burke GL, Hundley WG. Anthracycline-associated T1 mapping characteristics are elevated independent of the presence of cardiovascular comorbidities in cancer survivors. Circ Cardiovasc Imaging 2016;9:e004325.

37. Neilan TG, Coelho-Filho OR, Shah RV, Feng JH, PenaHerrera D, Mandry D, Pierre-Mongeon F, Heydari B, Francis SA, Moslehi J, Kwong RY, Jerosch-Herold M. Myocardial extracellular volume by cardiac magnetic resonance imaging in patients treated with anthracyclinebased chemotherapy. Am J Cardiol 2013;111:717-22.

38. McDonald RJ, McDonald JS, Kallmes DF, Jentoft ME, Murray DL, Thielen KR, Williamson EE, Eckel LJ. Intracranial gadolinium deposition after contrastenhanced MR imaging. Radiology 2015;275:772-82.

39. Kanal KM, Butler PF, Sengupta D, BhargavanChatfield M, Coombs LP, Morin RL. U.S. Diagnostic Reference Levels and Achievable Doses for 10 Adult CT Examinations. Radiology 2017;284:120-33.

40. Cardiac computed tomography (CT) - FAQs for health professionals I IAEA [Internet]. Available online: https:// www.iaea.org/resources/rpop/health-professionals/ radiology/computed-tomography/cardiac-computedtomography

41. Thirup P. Haematocrit: within-subject and seasonal variation. Sports Med 2003;33:231-43

42. Chen L, Linden HM, Anderson BO, Li CI. Trends in 5-year survival rates among breast cancer patients by hormone receptor status and stage. Breast Cancer Res Treat 2014;147:609-16.

Cite this article as: Monti CB, Zanardo M, Bosetti T, Alì M, De Benedictis E, Luporini A, Secchi F, Sardanelli F. Assessment of myocardial extracellular volume on body computed tomography in breast cancer patients treated with anthracyclines. Quant Imaging Med Surg 2020;10(5):934-944. doi: 10.21037/qims.2020.04.05 